\title{
Cuando el pasado interpela
}

\section{When the past interpellates}

\author{
Angélica De Sena \\ Profesora, Centro de Investigaciones y Estudios Sociológicos/Universidad de Buenos Aires. \\ angelicadesena@gmail.com
}

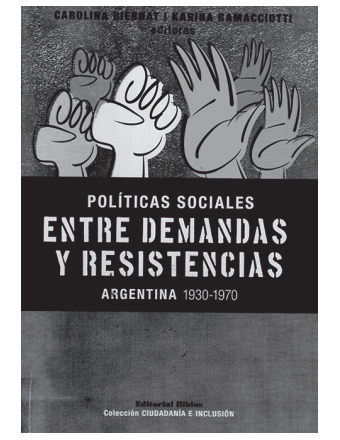

BIERNAT, Carolina; RAMACCIOTTI, Karina (Ed.). Políticas sociales: entre demandas y resistencias, Argentina, 1930-1970. Buenos Aires: Biblos. 2013. 208p.
T a presentación de un nuevo libro siempre es un evento festivo, L económico (nunca menor) y en este caso se agrega un tercer elemento: el resultado de un trabajo colectivo dando cuenta del esfuerzo que siempre significa y - también - la potencia de ello. En este libro interaccionan dos conceptos centrales: historia y políticas sociales.

Desde el sentido común construido, la historia suele presentarse como eventos del pasado relacionados con lo que "fue", con escaso vínculo con el presente. En tanto, aquello que se liga a "lo social" se instala como "lo bueno"; en cambio, "la política" adquirió cierta adjetivación no positiva. De este modo, el término social se conforma de cierta benevolencia y hasta condescendencia, ocluyendo sus funciones y otorgando una especie de amnesia de su génesis. Pero también es posible pensar en la historia como un conjunto de sucesos políticos, sociales, económicos, culturales, entre otros, de un pueblo en estricta relación con el presente. Ello permite revisar todas y cada una de las acciones del pasado en búsqueda de significaciones y comprensiones del presente (y posiblemente conjeturar el futuro). Por otro lado, al referirnos a las políticas sociales y efectuar cualquier tipo de análisis al respecto, es menester considerar el modelo de acumulación vigente. Por ello, en las sociedades capitalistas, el problema de la integración social se ubica en el centro de las discusiones respecto al orden social (Grassi, 2006; Castel, 1997, entre otros).

Existe cierto acuerdo en que la cuestión social, comienza a aparecer en el siglo XIX dando cuenta del riesgo de destrucción de la sociedad liberal, debido a las nuevas tensiones generadas por la industrialización. De este modo, la concepción de "lo social" es entendida en relación a los diferentes sistemas de regulación no relacionados con los del mercado, instituidos para intentar llenar la brecha entre lo político y lo económico (Hopp, 2011). En este punto, es necesario mencionar que las políticas sociales son un "conjunto de concepciones ideológicas que se plasman en diseños normativos e institucionales que buscan limitar las consecuencias sociales producidas por el libre juego de las fuerzas del mercado; concepciones que, al mismo 
tiempo, son útiles para construir legitimidad política" (Ramacciotti, 2010, p.193). Es decir que, las políticas sociales son "políticas" por tanto conllevan un conjunto de acciones, omisiones e intereses de múltiples actores que expresan necesidades políticas, económicas y sociales de un momento dado, en una construcción histórica y cultural que determina un terreno en el que convoca a las alianzas y las luchas dando paso a definiciones sobre la naturaleza de las intervenciones sociales como la consecuente inclusión de determinados núcleos poblacionales y la marginación de otros (Ramacciotti, 2010).

El punto de partida para Danani (2009) es que las políticas sociales "hacen sociedad" ya que son "aquellas específicas intervenciones sociales del Estado que se orientan (en el sentido de que producen y moldean) 'directamente' a las condiciones de vida y de reproducción de la vida de distintos sectores y grupos sociales, y que lo hacen operando especialmente en el momento de 'la distribución secundaria del ingreso'” (p.11).

De este modo, el vínculo entre políticas sociales e historia resulta por demás evidente, y su estudio imprescindible para las ciencias sociales; en ello se comprometen los distintos autores del libro que aquí se reseña. Presentación que se elabora con el objetivo de mirar hacia el pasado (a través de los artículos) mostrando cómo las lecturas del ayer se reflejan en el hoy; de este modo, la historia se manifiesta como "hechos", cargada de vida, sin ocultar las dificultades que conllevan el "mirar hacia atrás desde hoy", el analizar hechos como parte de procesos desde el presente, dejando en evidencia la complejidad de la(s) historia(s), interpelando los hilos de una trama que entrelazan los procesos sociales.

El título el libro y los años de referencia (1930-1970) muestran el fuerte peso del peronismo en la historia argentina y ello significa siempre tomar alguna posición dado que dicho movimiento generó (y genera) distancias y cercanías, amores y odios. Al mismo tiempo que deja entrever que "el peronismo" sigue siendo un renovado espacio de análisis historiográficos y sociológicos. El debate se abre con una introducción de Carolina Biernat y Karina Ramacciotti, siendo interpelado por las intervenciones sociales del Estado, en tanto este es un ámbito atravesado por una gran diversidad de intereses. Las editoras, junto al resto de los autores, toman como objeto de estudio al Estado, por donde circulan al menos tres grandes interrogantes ¿qué son las intervenciones sociales del Estado?, ¿cuáles son las políticas sociales?, ¿cómo es el vínculo entre Sociedad-Mercado-Estado?, mostrando la cuestión social como arena de la acción política. De este modo, se tejen conceptos tales como: acción/omisión; inclusión/ exclusión; marginación/subordinación; caridad/justicia social; estallido/conflicto social; demandas particulares/presiones sociales. Así, la cuestión social se observa en relación con las condiciones de vida de las personas (salud, educación, vivienda, alimentación, seguridad), el rol de los distintos actores sociales (iglesia, partidos políticos, empresarios, sindicatos, ONGs) y el lugar de una multiplicidad de excluidos (viejos, niños, prostitutas, locos, presos, desviados sociales) que se relacionan en la intersección del Estado-Mercado-Sociedad.

El primer artículo del libro es de Adrian Cammarota, titulado "Juventud, género y educación en la mira del primer peronismo", basa su análisis en la observación del periódico estudiantil de una escuela secundaria de la ciudad de Morón (provincia de Buenos Aires), en la década del '40. El escrito se funda en las siguientes preguntas: ¿cómo era vislumbrada la adolescencia durante este período?, ¿qué tipo de asociacionismo juvenil eran los clubes colegiales?, ¿qué tipo de actividades se realizaban dentro de ese espacio?, ¿qué significaciones e 
imaginarios proyectados por la pedagogía escolar podemos hallar en las subjetividades de esos jóvenes? De este modo, observa a los adolescentes y jóvenes como sujetos de derechos según la reforma educativa en la Argentina, la intromisión del plano normativo y los programas escolares basados en tres pilares: equidad/género/participación democrática. Así, la institución escolar aparece como un espacio de formación complejo, habitado por la pugna de sentidos que involucra la conformación de "criterios de normalidad".

El siguiente artículo de María Ortiz Bergia "Asistencia benéfica y gobierno peronista en Córdoba", se inserta en el complejo universo de las organizaciones de la sociedad civil mostrando la coexistencia e interacción de estas y el Estado en el campo de las políticas sociales. Con las distintas denominaciones (nunca ingenuas), las asociaciones de beneficencia, las ONGs, las organizaciones de la sociedad civil, permiten elaborar un triángulo junto al Estado y el mercado en estrecha interacción para la "protección social" y "distribución del bienestar". El artículo muestra claramente cómo en esos procesos se intersecan concepciones respecto a qué es lo social y las ambiciones/intereses en disputa. La mirada de la gestión sanitaria ubica en disputa médicos y damas de beneficencia, abriendo el viejo espacio reducido a estas últimas, a cierta profesionalización y dejando (al lector) al menos dos preguntas ¿qué disputaban?, ¿sigue en vigencia el modelo de "ayuda" de "las damas"? La incorporación de los distintos actores e intereses en la arena de las políticas sociales durante los años 1940, no sólo las organizaciones de beneficencias, sino también los sindicatos, el Estado, la Fundación Eva Perón. En este sentido, se evidencia cómo los distintos lugares tienen algún dispositivo y alguien que se ocupa de "lo social".

El libro continúa con el trabajo de Marcelo Jerez "Política de vivienda en Jujuy durante el primer peronismo", una cuestión que refiere al pasado y al más brutal presente: el derecho a la vivienda para los estratos sociales bajos y medios. El período considerado, presenta una ampliación de la injerencia estatal, la cuestión de la vivienda figuró en el discurso oficial y fue incorporada en la reforma constitucional de 1949. El artículo muestra la cuestión habitacional en relación a los movimientos demográficos y urbanos e interpela respecto a la actualidad del tema en relación a la pobreza.

El cuarto artículo, de Jeremías Silva, "Formación de los agentes penitenciarios durante el peronismo", provoca la pregunta sobre la relación entre seguridad y políticas sociales pero también muestra cómo las problemáticas carcelarias, el castigo y la disciplina son parte la agenda pública. El artículo hace foco en los distintos actores y manifestaciones de la problemática carcelaria y las decisiones políticas en relación a la calidad e índole de los servicios y la profesionalización de los agentes. En tanto, deja entrever las cuestiones de corrupción que rondan el tema permitiendo interpretar que luego todo fue peor.

Claudia Daniel, en su artículo "Las estadísticas en la construcción del Estado social (1930-1943)", trabaja sobre una cuestión casi tan antigua como el Estado mismo. Entre los antecedentes de las estadísticas, sólo por mencionar algunos, se conocen en Cerdeña vestigios en piedras que se suponen son para contabilizar el ganado en el año 1000 a.C., del mismo modo, los egipcios realizan censos al igual que en China. Se considera que Sócrates, Herodoto y Aristóteles incentivaron las estadísticas por su importancia para el Estado. Esto lo entendió muy bien el Imperio Romano quien recopiló datos sobre población, superficie y renta y luego mejoraron los formatos de censos bastante similares a los actuales, con el 
objeto de obtener información capaz de diseñar e implementar acciones desde el Estado. Es decir, la función de las estadísticas se halla en estricta relación con la estrategia de un Estado, como fuente de información para el diseño de las políticas públicas, por ello su relevancia. El artículo muestra su construcción en un período no sin complejidades en la historia argentina y unido a ello la necesidad de contar con instrumentos de recolección de la información en cuestiones laborales. En tanto, no es posible en Argentina, al mencionar las estadísticas, no referirse a la terrible y burda manipulación de las mismas desde el año 2007.

El libro finaliza con el artículo de Daniela Testa “¡SOS Vacunas! Tensiones entre Estado y sociedad civil (1957-1971)", que muestra la preocupación por erradicar la poliomielitis durante muchos años y cómo reaparece en 1984, 1991. La preocupación de "los Estados" por la interacción política y - una vez más - los distintos actores/beneficiarios/asistidos/ en el centro de los padecimientos. El escrito permite interrogarse por la actualidad de la y las enfermedades, por la construcción de las agendas públicas, y también de la investigación social. La carga de normalizaciones en acciones y en investigaciones, la escasa vigilancia epistemológica y como consecuencia la "invisibilización" de temas, problemas y sujetos.

En estos seis artículos y la introducción, se muestra un recorrido con tintes federales al observar distintos rincones de la Patria. Dejando en claro cómo a la pobreza se la relacionó con la moral, con los desvalidos y por lo tanto con las distintas formas de la asistencia social. No sólo como un elemento de control social o para desactivar el conflicto, sino como un modo de "rehabilitación social", así se fue consolidando una forma de mirar hacia el control/ normalización/disciplina que requiere de la "militancia caritativa" para la reinserción social (¿qué también involucra una fuerte advertencia para el presente?).

Retomando el inicio de la presente reseña y luego de la lectura del libro editado por Biernat y Ramacciotti, las políticas sociales son "políticas", que construyen sociabilidades dado que tanto las acciones como las omisiones de cada una de ellas, construyen sentidos y conforman sujetos individuales y sociales al mismo tiempo que se consolida un imaginario colectivo, las mismas fundan y consolidan vivencialidades y sensibilidades - sensu Scribano (2012) - que estructuran el tejido social, por ello lo imprescindible y urgente de su análisis revisando detenidamente sus diseños y sus consecuencias para no legitimar desigualdades y peores condiciones de vida para nuestros pueblos.

\section{REFERENCIAS}

CASTEL, Robert.

Las metamorfosis de la cuestión social: una crónica del Salariado. Buenos Aires: Paidós. 1997.

DANANI, Claudia.

La gestión de la política social: un intento de aportar a su problematización. In: Chiara, Magdalena; Di

Virgilio, María Mercedes (Org.). Gestión de la política social: conceptos y herramientas. Buenos Aires: Prometeo. p.25-51. 2009.

GRASSI, Estela.

Integración y necesidades sociales: reflexiones desde el punto de vista de la igualdad. Trabajo presentado en 3. Congreso Argentino de Política Social, 2006. Buenos Aires. 2006.
HOPP, Malena.

Relación Estado-sociedad civil en las políticas de desarrollo socio-productivo en Argentina contemporánea. Revista Katálysis, v.14, n.1, p.13-22. 2011.

RAMACCIOTTI, Karina.

Reflexiones en torno a cómo pensar las intervenciones sociales del Estado. Revista de Estudios Marítimos y Sociales, v.3, n.3, p.193-193. 2010.

SCRIBANO, Adrian.

Sociología de los cuerpos/emociones. Revista Latinoamericana de Estudios sobre Cuerpos, Emociones y Sociedad, v.4, n.10, p.91-111. 2012. 